\title{
On-demand and Greedy Forwarding Based Routing Protocol for Mobile Ad Hoc Networks
}

\author{
Jinke Huang ${ }^{1, a}$, Xiaoguang Fan $^{1, b}$, Xin Xiang $^{1, c}$ and Jian Xiong ${ }^{1, d}$ \\ ${ }^{1}$ Aeronautics and Astronautics Engineering College, Air Force Engineering University, \\ Xi'an, 710038, China \\ a86297609@qq.com, b15829717996@163.com, c460667937@qq.com, dxj20111799@163.com
} Keywords: mobile ad hoc networks, routing protocol, scoped flooding, mobility prediction, delayed
route requests, performance evaluation.

\begin{abstract}
The dynamic topology of a mobile ad hoc network poses a real challenge in the design of routing protocol, especially in high-dynamic environments. In this paper, we proposed a new routing protocol which based on on-demand and greedy forwarding. This protocol proposed three opinions to RGR, i.e., through scoped flooding to decrease control overhead in route discovery phase, through mobility prediction to monitor the condition of reactive path and help nodes to choose the proper next-hop in GGF phase, and through delayed route requests to reduce unnecessary waste of network resources. Compared to RGR, AODV, Modified-RGR and Optimized-RGR, simulation results show that the improved RGR not only have high packet receive ratio, but have low average normalized routing overhead and average end-to-end delay.
\end{abstract}

\section{Introduction}

As a self-organizing, multi-hop and does not depend on ground infrastructure wireless network, MANETs (Mobile Ad Hoc Networks) has got the concerns in industry. However, it is challenging to design the routing protocol of MANETs, which the characteristics of dynamic topology, no center, connectivity restrictions, and limited bandwidth, namely it is hard to design an effective and reliable routing protocol to ensure network connectivity, effectiveness and utilization of resources ${ }^{[1-2]}$.

Depending on updating mechanism, routing protocols can be divided into active (table-driven) routing, reactive (on-demand) routing, mixed routing and geographic routing. Active routing needs to periodically broadcast routing information to actively maintain routes, and periodic routing information exchange needs to consume a lot of bandwidth resources, so this strategy is applicable to smaller networks; reactive routing does not need to periodically broadcast routing information, the routing discovery process would be performed only when the source and destination need to communicate but the link is not known to the source node. The route discovery can cause a certain delay, so the protocol is applicable to smaller networks. Mixed routing is a protocol, which combines the active and reactive routing, that is, within a certain zone using active or reactive strategy, without the zone it uses reactive or active strategy. This strategy is mainly designed to large-scale, highly dynamic MANETs; geographical routing is coming and developing with the development of GPS, the node equips positioning device nodes can accurately get their own geographical location and timing, according to them, the source node can be "purposely" transmitted the packets to the destination direction and ultimately reach the destination node.

In the high dynamic environment, the simple use of reactive or geographical routing has been unable to meet the performance needs of user. Therefore, literature [3] proposed a new route pattern: the "Reactive -Greedy-Reactive" (RGR), which enables nodes adapted to high dynamic scenes very well. However, due to its control messages (such as RREQ) is broadcast in the entire network, the control overhead will be relatively high. Literature [4], which depends on RGR, uses the scoped flooding and mobility prediction mechanisms to increase the packet receive ratio and reduce the end to end delay in a certain extent, but the decline of control overhead is not good. Literature [5] , which depends on RGR, uses the scoped flooding and delayed new routs establishing to reduce the control 
overhead, but packet receive ratio and average end to end delay are not good. Literature [6] proposed a modified RGR, which added stability metric link in the routes establishing process to RGR protocol. The Optimized-RGR ${ }^{[7]}$ is a protocol which introduced greedy geographic forwarding (GGF) repair strategy to Modified-RGR. Literature [6] and [7] generally increased the packet receive ratio, but cannot ensure the network have lower control overhead and average end-to-end delay.

In order to improve the overall performance of RGR network, this paper puts forward three optimization opinions to RGR in high dynamic environment, namely: 1) scoped flooding strategy: using the rate and direction of destination to provide more precise location information for intermediate nodes to "purposely" restricts the flooding area to reduce control overhead in routs discovery phase; 2 ) mobility prediction strategy: on one hand, to monitor the state of reactive paths; on the other hand, to help nodes select the appropriate node as the next-hop in GGF phase; 3) delayed routes request strategy: when the existing path fails, delayed transmission of RERR to delay the establishment of new routes to reduce the unnecessary waste of resources.

Simulation results showed that: the packet receive ratio, average routing overhead and average end-to-end delay of improved RGR protocol (Improved-RGR) is outperformed the RGR, AODV, Modified-RGR and Optimized-RGR.

\section{Basic theory}

\subsection{RGR protocol}

RGR protocol absorbed the characteristic of topology-based and location-based routing protocol, and can suitable to high dynamic environments. Traditional, RGR combines the AODV protocol and GGF strategy, that is, when the reactive routing is failure, the network will use the GGF mode to transmit; when one of the two conditions take place, namely: 1) When the live time of data packet (TTL) decreases to zero; or 2) GGF mode cannot help nodes find a relayed node in the direction of destination, it will abandon the GGF to use reactive strategy.

The Innovation of RGR protocol is: reactive and GGF strategy can be used to transmit data packets. Compared to pure geographic routing, RGR protocol does not require a special location service mechanism to provide location information of the destination but use reactive strategy instead. Compared to pure reactive routing protocol, RGR protocol does not require routs repaired process to mend the disconnected routes but use GGF strategy instead.

\subsection{Scoped flooding based protocol}

DREAM (Distance Routing Effect Algorithm for Mobility) is a directional flooding routing protocols. It stipulates that in data transmitting phase, the source first uses location information to calculate the fan-shaped sector to the destination, and then the source node forwards the packet to all its one-hop neighbors in this sector. When the neighbor receives the packet, it will continue to flood the packet until the packet arrives at the destination.

LAR (Location-Aided Routing) ${ }^{[8]}$ protocol uses geographic information of the destination to limit the flooding area, somewhat similar to the DREAM protocol. Its scoped flooding mechanism, which is used to reduce the cost of control, is the most commonly used method. In AODV and DSR (Dynamic Source Routing) protocol, the geographic location information of the destination cannot be used directly ${ }^{[9]}$. LAR uses AODV or DSR protocol to associate the destination and the corresponding location information. LAR protocol only floods the RREQ packet to the area not the entire network, which contains the destination node. In the path repairing phase, each intermediate node will compare its own location with the specific location search area included in RREQ packet. If the intermediate node is in this area, then continuing forwarding RREQ, otherwise discarded it ${ }^{[10]}$. If the node, which is on path leading to the destination, is outside the search area, LAR will re-flooding RREQ packet. 


\subsection{Mobility prediction based protocol}

Literature [11-12] proposed many methods to talk about how to use mobility prediction mechanism in the reactive protocol. But most of the mechanisms are to find the most stable path as the best path within the known path. Literature [11] proposes to use the Link Expiration Time (LET) to optimize the routing protocol. This strategy estimated the LET through nodes' location information within the control packet. And then add it to the RREQ packet. After that, the intermediate nodes will broadcast RREQ packets to all of its neighbors. When the destination receives the RREQ packet, it can master the survival time of all the links. By finding the smallest link path, the destination can learn the Route Expiration Time (RET), so the source can find the most stable Link to transmit data.

MPRP (Mobility Prediction Routing Protocol) ${ }^{[12]}$ predicts link status in the data transmitting phase, and encapsulates location information in data packets. In data transmitting phase, the intermediate can learn the location information from the node, which forwards data packet to it. By comparing the location information extracted from two successive the received packets, the current node can determine the remaining duration of the link and eliminate some unnecessary intermediate nodes. This mechanism is simple and does not require complex calculations and packet formats. However, it must be added a prediction table to storing prediction information, and added a new Route Expired (REXP) packet to informing paths' status to current node. This thesis will find a similar mechanism to replace the packet in RGR.

\section{Improved-RGR}

\subsection{Scoped flooding mechanism}

To reduce the number of RREQ packets to decrease the routing overhead and avoid network congestion, this thesis will propose a new scoped flooding mechanism.

First, it is assumed that not only the source but also other nodes in the network can learn the location information of the destination. When finding the routes for the first time, the distance between source and destination will be set to 0 and encapsulated in the RREQ packet. Then, the source broadcasts the RREQ packet to its neighbors. When its neighbors receive the packet, they will check whether the packet has the location information of destination or not. If not, the neighbors continue broadcasting received RREQ packet. Otherwise, the neighbors (intermediate forwarding node) will calculate the distance between itself and destination, and compare the distance values within the RREQ. If the value, extracted from the RREQ packet, is 0 (i.e., this node does not know the location information of destination), it will be replaced by the value that between intermediate node and destination. If not, the intermediate will compare it with the value within the RREQ packet. When the value is less than the originally carried by RREQ, the value will be replaced by the newly calculated distance value, and rebroadcast together with the RREQ packet. Otherwise, intermediate will discard the RREQ packet. This process will be continued until the RREQ packet arrived at the destination.

When no nodes know the location information of destination, Improved-RGR will use the same mechanism like RGR. When the source uses inaccurate location information for routing, the scoped flooding mechanism will also fail. In this case, the source will rebroadcast RREQ packet after a period of unnecessary waste of time.

\subsection{Mobility prediction mechanism}

In RGR protocol, data packets can be transmitted to the destination when reactive path exist. In data transmitting phase, the intermediate will learn the state of next-hop through two successive HELLO packets. If the intermediate does not receive 3 consecutive HELLO packets, it is considered that the link is disconnected. Thereafter, the data will be forwarded through GGF mode. HELLO 
packets are broadcasted once per second, so in this mechanism the link is disconnected when it delayed 2 to 3 seconds. Therefore, the intermediate cannot immediately detect the link is disconnect. In this phase, the intermediate will still use reactive mode to data transmission. Thus, these data packets will be lost, and will not be regained through GGF mode.

But it can be replaced by reconfiguring the link standards, such as it is assumed that the link is disconnected when the intermediate cannot receive 1 HELLO packet to reduce packet loss. Although this mechanism can shorten the disconnected link discovering time, it will be lead to more RRER sending, which in turn may lead more HELLO packets conflict. If reduce the frequency for HELLO packets sending, it will generate more HELLO packets in limited time when there are more nodes in the network, and hence increase the control overhead.

To solve this problem, the proposed mobility prediction mechanism will use a velocity vector with $t_{-}$stamp before sending packets, which is part of the periodic HELLO packet, to calculate the distance between the current node and the next-hop. When the next-hop node is beyond the communication range of the current, this disconnection will be aware immediately, and then use the GGF to avoid data packets losing.

In this prediction mechanism, beside RREQ, RREP and HELLO packets need to carry more information, but other control messages are similar with RGR. In route discovering phase, RREQ and RREP packet will not only carry the location information of destination, but also piggyback its one-hop nodes' speed, direction and timestamp. In route maintenance phase, nodes periodically broadcasts HELLO packets, which contain its own position, speed, direction and time stamp information, to inform its neighbor nodes. And this information will also be recorded by the intermediate.

When the path discovering process is finished, the source will send the cached data to destination. Not like RGR, it is first to estimate the distance between current node and the next-hop. Namely, using of equation (1), current node will estimate of the distance to its next-hop.

$$
\begin{aligned}
& X_{p}=X_{n}+V \times \cos (\theta) \times\left(c_{-} \text {time }-t_{-} \text {stamp }\right) \\
& Y_{p}=Y_{n}+V \times \sin (\theta) \times\left(c_{-} \text {time }-t_{-} \text {stamp }\right)
\end{aligned}
$$

In equation (1), $X_{p}$ and $Y_{p}$ is the predicted location information of next-hop; $X_{n}$ and $Y_{n}$ is the location information before prediction, $t_{-}$stamp records the time of this node in this position. $v$ and $\theta$ denote the speed and direction information of next-hop node. These parameters can be extracted from the current node's routing table.

Using equation (2), it can be determined that whether the next-hop node is within communication range of the current node.

$$
D_{n}=\sqrt{\left(X_{o}-X_{p}\right)^{2}+\left(Y_{o}-Y_{p}\right)^{2}}
$$

In equation (2), $D_{n}$ is the actual distance between current node and the next-hop. $X_{o}$ and $Y_{o}$ is current node's location information.

If $D_{n}$ is smaller than the transmission range of the current, the current continues to transmit data to the next-hop through the reactive mode. If not, the current node will stop sending data immediately to use GGF mode. In GGF mode, the current node can learn the network topology information via the same prediction mechanism. And then it will send the data to the closest node of destination.

\subsection{Delayed route request mechanism}

In RGR protocol, if data transmission through the reactive mode is fail, the network will start GGF to continue to forward data. And it can be ensured that the data can be transmitted to the nearest node of destination through GGF. Moreover, the node which on the disconnected path will immediately send RERR packets along the opposite path until it reaches the source to inform the reactive is fail. At this time, if the source continues to send data to the same destination, the path repairing process must be started. 
In this mode, when a path is disconnected, each intermediate node still has the ability to continue to use the GGF mode to transmit data to destination even if there is not available reactive path to destination. So, it is not necessary to send RERR packets immediately. Furthermore, the literature [13] pointed out that when there are fewer hops between the current node and destination, it is high probability to use GGF to transmit data to destination.

Before the new reactive path established, the intermediate node will continue to send packets to its closest neighbor of destination. Here, RERR packet will be send after a period of delay (typically sending a HELLO packet interval) along the opposite. When RERR packet reached the source, the new path repairing process will be started. In high dynamic environment, this mechanism will reduce the total number of RREQ packets.

\section{Performance evaluation}

\subsection{Configuration}

In this paper, we use NS2 to analyze Improved -RGR protocol. In simulation, each node is randomly distributed in $300 \times 300 \mathrm{Km}$ area, and moved according to random waypoint model. Transmission of each node is $15 \mathrm{Km}$, velocity evenly distributed between $300 \sim 1100 \mathrm{~m} / \mathrm{s}$ and the simulation time is $30 \mathrm{~min}$.

The simulation model of each node is the same. The physical layer uses the free space model. Medium access control (MAC) layer uses TDMA (Time Division Multiple Access) protocol. Network layer uses the Improved-RGR protocol. Application layer uses the constant bit rate service (CBR) model. All network members generate new services with the same probability, and the duration is subject to negative exponential distribution, and the packet length is to the Poisson distribution. The channel ratio is $5 \mathrm{Mbit} / \mathrm{s}$, and the packet size is $1024 \mathrm{Kbyte}$.

\subsection{Simulation results and analysis}

To analyze the performance of Improved-RGR routing protocols, this paper compared it with RGR, AODV, Modified-RGR and Optimized-RGR.

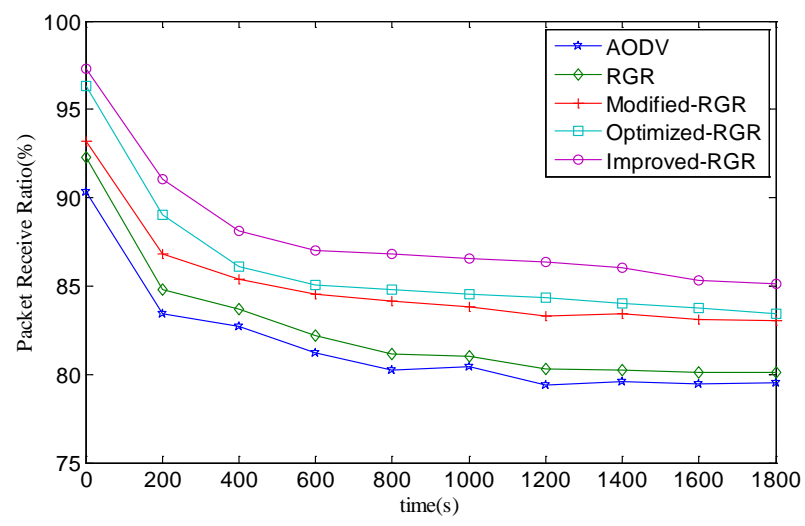

Fig.1 Packet Receive Ratio

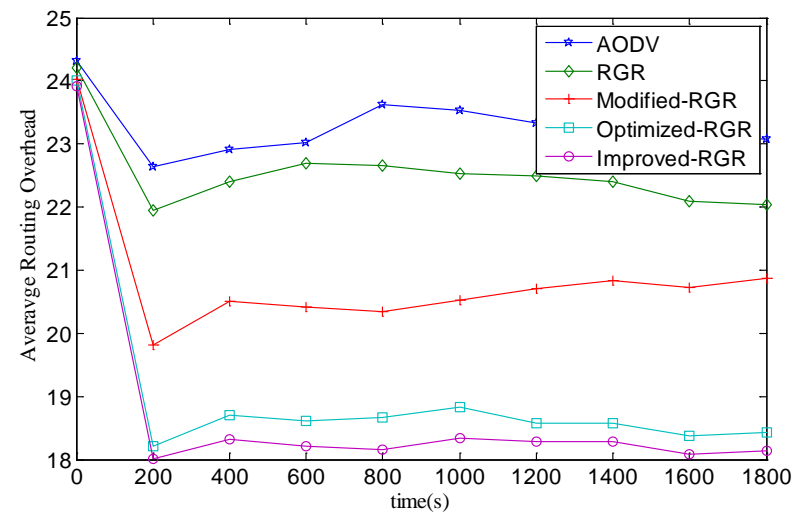

Fig.2 Average Routing Overhead

Fig.1 depicts the packet receive ratio of the five protocols. From the figure, it can be seen from the figure that the ratio of Improved-RGR is the highest, followed by the Modified-RGR and Optimized-RGR, and RGR and AODV protocol has little difference. This is because the RGR and AODV is no real-time detection of reactive link status. The Modified-RGR, Optimized-RGR and Improved-RGR protocol is capable of detecting link status, so when the link is disconnected they can use GGF mode to forwarding data. And compared to Modified-RGR and Optimized-RGR, Improved-RGR protocol can select the best next-hop node and have a high packet receive ratio.

Fig. 2 depicts the average routing overhead of the five protocols. From the figure, it can be seen that the average routing overhead is high at the beginning. However, with the communication links 
establishing, the demand for control messages is reduced, so the average routing overhead decreased, but it fluctuate only within a limited range. And the average routing overhead of Improved-RGR is the minimum, this proved that the Improved-RGR protocol successfully exploited the scoped flooding and delayed route request mechanism to reduce the number of RERR and RREQ packets, and hence reducing the average routing overhead and avoiding waste of bandwidth resources.

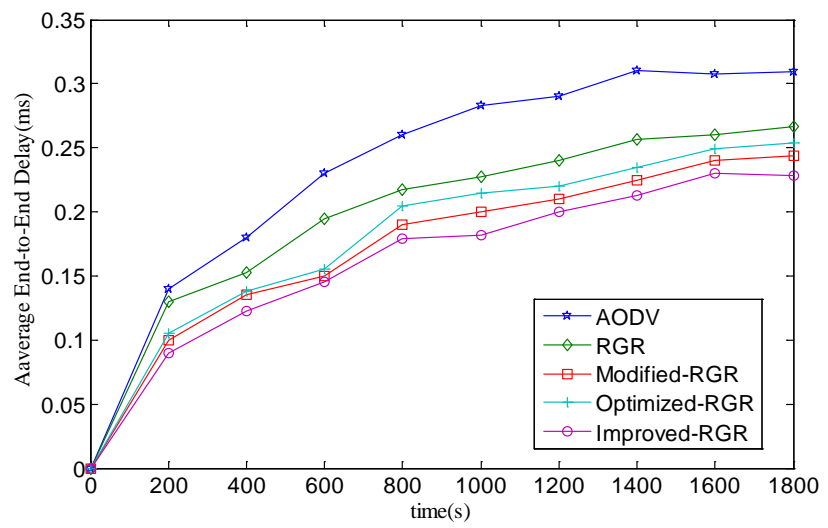

Fig.3 Average End-to-End Delay

Fig.3 depicts the average end-to-end delay of the five protocols. From the figure, it can be seen from the figure that the average end-to-end delay of AODV is the highest, followed by the RGR, and the average end-to-end delay of other protocols are similar. But Improved-RGR is slightly less than the Modified-RGR and Optimized-RGR. This is because the Improved-RGR not only reduces the number of control packets to some extent to alleviate network congestion, but help nodes choose the appropriate next-hop node to relay. As a result, the average end-to-end delay of Improved-RGR is minimal.
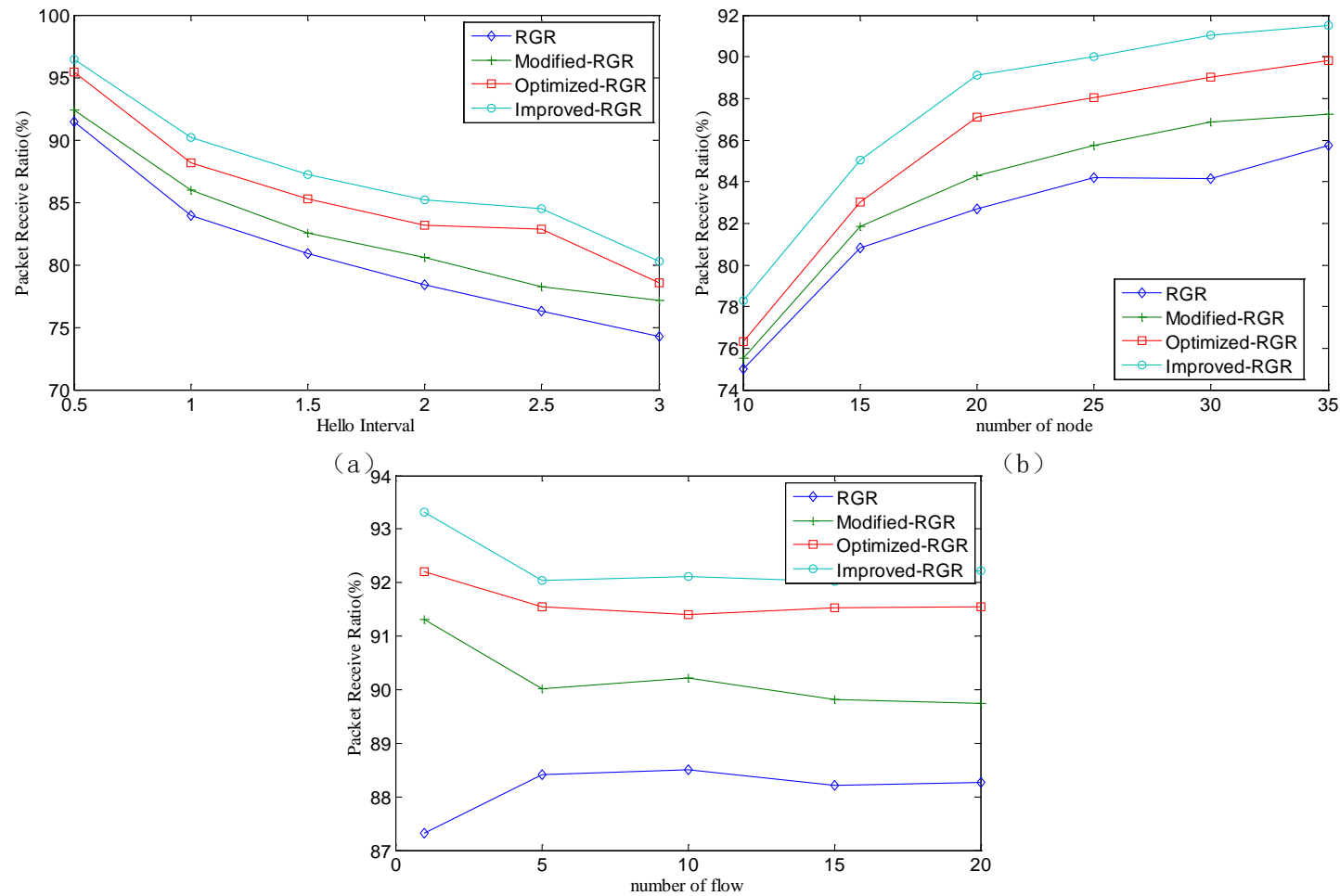

(b)

(c)

Fig.4 Packet Receive Ratio

Fig.4 (a) reflects the impact of HELLO packet interval to packet receive ratio. It can be seen from the figure, when the interval increases, the packet receive ratio of the four will fall. Thanks to GGF repair strategy, Optimized-RGR is higher than Modified-RGR at the same HELLO packet interval. But Improved-RGR uses the mobility prediction mechanism to select the best node to next-hop to greatly reduce the probability of failure for path finding, so its packets receive ratio is the highest. 
When HELLO packet transmission interval is shorter, the ratio will be higher, but control overhead will be greater. Therefore, HELLO packet interval should considered the affordable control overhead and requirements for packet success ratio. When HELLO packet interval is $0.5 \mathrm{~s}$, the ratio of four protocols is the highest. When HELLO packet interval is 1s, their difference is small and the packet success ratio of Improved-RGR is still maintained at a high level (greater than 90\%). Therefore, considering the control overhead and packet receive ratio, Improved-RGR protocol selected the HELLO packet delay and route request delay is 1s. Fig.4 (b) reflects the impact of number of nodes to packet receive ratio. It can be seen from the figure, when the number of nodes is small, the network is relatively sparse, the data packet transmission requirements are low, so the packet receive ratio of the four protocol is almost close. As the number of nodes increases, their packet receive ratio will increase, but Improved-RGR is always highest, which should be attributed to its scoped flooding and mobility prediction mechanism helps node selects the optimal next-hop forwarding node, which is the closest to destination. Fig.4 (c) reflects the impact of data traffic to packet receive ratio. It can be seen from the figure, the packet ratio of Improved-RGR is the highest, and although from 1 to 5, its packet receive ratio drops quickly, but from 5 on it stabilized. Moreover, its trend is similar to Modified-RGR Protocol.

\section{Summary}

In order to improve the performance of RGR protocol in high dynamic environment, this thesis uses scoped flooding, mobility prediction and delayed route requests to improve it. And simulation results showed that Improved-RGR is outperformed the RGR, AODV, Modified-RGR and Optimized-RGR, namely, the Improved-RGR not only increase the packet receive ratio, but also reduce the average control overhead and average end-to-end delay.

\section{Acknowledgments}

The communicated author is Jinke Huang, and his Email is 86297609@qq.com.

\section{References}

[1] Linnan H. Medium access control protocols for wireless mobile ad hoc networks[C]//2005 Asia-Pacific Microwave Conference Proceedings, Shanghai: IEEE Press, 2005. $4 \sim 7$.

[2] Swidan A, Khattab S, Abouelseoud Y and Elkamchouchi H. A secure geographical routing protocol for highly-dynamic aeronautical networks[C]// MILCOM 2015-2015 IEEE Military Communications Conference, Tampa, FL: IEEE Press, 2015. 26 28.

[3] Rostam S, Marc S H, Thomas K, Yifeng Z, Jun L and Louise L. Combined Reactive-Greedy-Reactive Routing for Unmanned Aerial Ad-hoc Networks [C]// 2012 8th International Wireless Communications and Mobile Computing Conference (IWCMC). Limassol: IEEE Press, 2012: 820 826.

[4] Yi L, Marc S H, Thomas K. Enhancements to Reduce the Overhead of the Reactive-Greedy-Reactive Routing Protocol for Unmanned Aerial Ad-hoc Networks [C]// 2012 8th International Conference on Wireless Communications, Networking and Mobile Computing (WiCOM). Shanghai: IEEE Press, 2012: 1 4.

[5] Yi L, Marc S H, Thomas K. Improving Routing in Networks of UAVs via Scoped Flooding and Mobility Prediction [C]// 2012 IFIP Wireless Days (WD). Dublin: IEEE Press, 2012: 1 6.

[6] Jean-Daniel Medjo Me Biomo, Thomas K, Marc S H. Routing in Unmanned Aerial Ad hoc Networks: A Recovery Strategy for Greedy Geographic Forwarding Failure [C]// 2014 IEEE 
Wireless Communications and Networking Conference (WCNC). Istanbul: IEEE Press, 2014: 2236 $\sim 2241$.

[7] Fraser C, Kevin C, Jose S, and Sandra M. A Survey of Geographical Routing in Wireless Ad-Hoc Networks [J]. IEEE Communications Surveys \& Tutorials, 2012, 15(2): 621 653.

[8] Mukesh G. A modify the directional aware nodes using LAR Routing Protocol \& GPS technology in MANET[C]// 2013 International Conference on Green Computing Communication and Conservation of Energy (ICGCE), Chennai: IEEE Press, 2013. $909 \sim 913$.

[9] Liu J, Li F M. An Improvement of AODV Protocol Based on Reliable Delivery in Mobile Ad Hoc Networks[C]// 2009. IAS '09. Fifth International Conference Information Assurance and Security, Xian: IEEE Press, 2009. 18 20.

[10] Catia Valdman, Marcello L R de Campos, José Antonio Apolinario. A geometrical stopping criterion for the LAR algorithm[C]// 2012 Proceedings of the 20th European Signal Processing Conference (EUSIPCO), Bucharest: IEEE Press, 2012. 2104 2108.

[11] Hasan Abdulwahid, Benxiong $\mathrm{H}$ and Zijing C. Energy-Efficient and Reliable Routing for Mobility Prediction-Based MANETs[C]// 2015 11th International Conference on Mobile Ad-hoc and Sensor Networks (MSN), Shenzhen: IEEE Press, 2015. $43 \sim 51$.

[12] Lin L, Qibo S, Shangguang Wang and Fangchun Y. A geographic mobility prediction routing protocol for Ad Hoc UAV Network[C]// 2012 IEEE Globecom Workshops, Anaheim, CA: IEEE Press, 2012, 1597 1602.

[13] Rostam S, Marc S H, Thomas Kunz, Yifeng Z, Jun L and Louise L. The performance of Greedy Geographic Forwarding in Unmanned Aeronautical Ad-Hoc Networks [C]// 2011 Ninth Annual Communication Networks and Services Research Conference (CNSR). Ottawa, ON: IEEE Press, 2011: $161 \sim 166$. 